\title{
The association between coenzyme $Q 10$ concentrations in follicular fluid with embryo morphokinetics and pregnancy rate in assisted reproductive techniques
}

\author{
Süleyman Akarsu ${ }^{1}$ • Funda Gode ${ }^{1}$ - Ahmet Zeki Isik ${ }^{1} \cdot$ Zeliha Günnur Dikmen $^{2}$. \\ Mustafa Agah Tekindal ${ }^{3}$
}

Received: 27 November 2016 / Accepted: 24 January 2017 /Published online: 9 February 2017

(C) Springer Science+Business Media New York 2017

\begin{abstract}
Purpose This study seeks to evaluate the association between follicular fluid (FF) coenzyme Q10 (CoQ10) levels, embryo morphokinetics, and pregnancy rate.

Methods Sixty infertile patients who underwent intracytoplasmic sperm injection (ICSI) cycles were included in the study. For each patient, CoQ10 level of the follicular fluid was measured by high-performance liquid chromatography system. After the ICSI of each oocyte, the relationship between the level of CoQ10 content of each follicular fluid, the subsequent embryo quality, and embryo morphokinetics was investigated. The relationship between the level of CoQ10 content of each follicle and optimal time-lapse parameters for the embryos of these follicles including $\mathrm{t} 5, \mathrm{~s} 2$, and $\mathrm{cc} 2$ was also analyzed. The embryos were further classified into four categories, namely, grades $\mathrm{A}, \mathrm{B}, \mathrm{C}$, and $\mathrm{D}$, according to morphokinetic parameters using t5-t2 and t5-t3 (cc3). Each follicular fluid analysis was performed for a single oocyte of a single embryo which was transferred to the patients. Additionally, follicular fluid CoQ10 levels and pregnancy rates were evaluated.

Results Follicular fluid CoQ10 levels were significantly higher in grades $\mathrm{A}$ and $\mathrm{B}$ than grades $\mathrm{C}$ and $\mathrm{D}$ embryos $(p<0.05)$. The concentration of CoQ10 levels was
\end{abstract}

The original version of this article was revised: Mustafa Agah Tekindağ was corrected to Mustafa Agah Tekindal.

Funda Gode

funda.gode@gmail.com

1 In Vitro Fertilization Centre, Izmir Medical Park Hospital, Karsiyaka, Turkey

2 Department of Medical Laboratory, Faculty of Medicine, Hacettepe University, Ankara, Turkey

3 Department of Biostatics, Izmir Medical Park Hospital, Izmir, Turkey significantly higher in the pregnant group $(p<0.05)$. There was no significant correlation between optimal t5 and s2 morphokinetic parameters and CoQ10 levels. However, CoQ10 levels were significantly higher in follicular fluid of embryos which had optimal cc2 $(p<0.05)$.

Conclusion High follicular fluid CoQ10 level is associated with optimal embryo morphokinetic parameters and higher pregnancy rates.

Keywords Coenzyme Q10 · Embryo morphokinetics · Follicular fluid $\cdot$ Pregnancy rate $\cdot$ Assisted reproduction

\section{Introduction}

Oocyte quality remains the most important issue during in vitro fertilization (IVF) cycles. The main factors affecting the oocyte quality are the age of the women and the status of ovarian reserve. The unfortunately age-related decline in oocyte quality is a major difficulty in the treatment of older patients. The main reason for this decline was related to an accumulation of point mutations and deletions of mitochondrial deoxyribonucleic acid (DNA) $[1,2]$. Oocyte maturation is a complex process including nuclear and cytoplasmic components and it needs an accurate meiotic spindle. Mitochondria is the key regulator of energy production via oxidative phosphorylation which is extremely important in oocyte maturation process. Any problem during oxidative phosphorylation might affect both the quality and DNA content of the oocytes. Thus, the state of energy production and mitochondrial membrane potential of oocytes were found to be related to the implantation potential in previous reports [3-5].

Oxidative phosphorylation involves the action of the mitochondrial respiratory chain which consists of four complexes that are located in the inner mitochondrial membrane. 
Complexes I and II oxidize products of the tricarboxylic acid and transfer the electrons to coenzyme Q10 $(\mathrm{CoQ})$. The CoQ10 transfers the electrons to complex III (cytochrome reductase); the electrons are then transferred to complex IV which reduces $\mathrm{O}_{2}$ to produce $\mathrm{H}_{2} \mathrm{O}$. These steps result in adenosine triphosphate (ATP) production by complex V $[6,7]$. Therefore, CoQ10 has a critical role in cell growth and energy metabolism. One of the most important factors during oogenesis and oocyte maturation is energy consumption. Thus, CoQ10 was reported to improve development, hatching, cell proliferation, and ATP content of in vitro-produced bovine embryos [8].

Coenzyme Q10 is a widely used antioxidant supplement in several clinical conditions such diabetes, cancer, and cardiovascular diseases [9]. Also, it was shown that CoQ10 improves sperm motility, semen quality, and pregnancy rate $[10,11]$. Recently, Bentov et al. reported that the use of coenzyme Q10 supplementation resulted in reduced aneuploidy and increased pregnancy rates. However, the study was terminated before reaching the target population and the results were not statistically significant [12]. Gat et al. reported that additional treatment with CoQ10 increased the antral follicle count and ovarian response in patients with decreased ovarian reserve. However, there was no significant difference in clinical outcomes [13]. Although CoQ10 is widely preferred as a supplement in infertile patients, there is still a lack of evidence about the exact beneficial effects of CoQ10 on embryo quality and the pregnancy rates in humans.

The microenvironment of the oocyte is an important factor for the oocyte quality. Oocyte completes its maturation process in the follicular fluid (FF), thus, the biochemical characteristics of the FF might play a critical role in oocyte and subsequent embryo quality [14]. There is a bidirectional connection, including nutrient, oxygen, and many growth factors between oocyte and cumulus cells. The follicular content of these factors was related to the oocyte quality and pregnancy rates in previous reports [15].

Therefore, the principal goal of this study was to assess the relationship between follicular fluid CoQ10 levels, the subsequent embryo quality, and pregnancy rates. The relationship between follicular fluid CoQ10 levels and embryo morphokinetics was also evaluated.

\section{Materials and methods}

\section{Patients}

One hundred one patients with unexplained infertility who were admitted to the IVF center of Izmir University Medical Park Hospital were enrolled to this prospective study and 60 of them were included to the analysis. The allocation process is defined in detail in the "Patient and follicular fluid sample selection process" section further below. The study was approved by the Ethics Committee and Institutional Review Board of the Izmir University. Informed consent was taken from all of the patients. The study group consisted of patients with unexplained infertility. The exclusion criteria were patient's age $>41$, male factor, polycystic ovary syndrome, diminished ovarian reserve, endometriosis, the presence of hydrosalpinx and any endocrinological disease, patients who refused single embryo transfer and cases with no available good quality embryo for transfer with sampled follicular fluid.

Also, the serum levels of follicle stimulating hormone (FSH), estradiol (E2), and antimullerian hormone (AMH) were measured. And patients with $\mathrm{FSH}>1 \mathrm{mIU} / \mathrm{mL}$, $\mathrm{LH}>15 \mathrm{mIU} / \mathrm{mL}$, and $\mathrm{AMH}<1 \mathrm{ng} / \mathrm{mL}$ were excluded.

\section{Ovarian stimulation and intracytoplasmic sperm injection (ICSI)}

All patients underwent the antagonist protocol for ovarian stimulation. Daily recombinant gonadotropin (125-300 IU, Gonal-F, Merck, Turkey) doses were administered according to patient's age, ovarian reserve, and body mass index. When the leading follicle exceeded $14 \mathrm{~mm}$, a gonadotropin antagonist (Cetrotide $0.25 \mathrm{mg}$; Merck) was started and when at least two follicles reached $18 \mathrm{~mm}$, hCG (Ovitrelle $250 \mu \mathrm{g}$; Merck) was applied. The oocyte retrieval was performed at $36 \mathrm{~h}$ after triggering. After the oocyte retrieval, oocytes isolated from the samples of FF were evaluated separately from the other oocytes. Furthermore, cumulus oocyte complexes were then incubated for $2 \mathrm{~h}$ in Quinn's advantage medium (SAGE 1-step, Origio, Denmark) at $7 \% \mathrm{CO}_{2}$ and $37{ }^{\circ} \mathrm{C}$ before oocyte denudation. The cumulus cells were removed by mechanical pipetting in $10 \mathrm{IU} / \mathrm{mL}$ hyaluronidase (HYASE $10 \times$ Vitrolife, Sweden). Nuclear maturation was determined by the identification of the first polar body. Microinsemination was then performed in Quinn's advantage medium containing HEPES (SAGE Media, Origio, Denmark). Subsequently, oocytes were placed individually on a pre-equilibrated Embryoslide (Vitrolife, Denmark) containing 12 wells filled with an overlay of $1.2 \mathrm{~mL}$ mineral oil (Origio, Denmark). The oocytes were incubated in the time-lapse monitoring system (Embryoscope; Unisense FertiliTech, Denmark) in an atmosphere of $5 \% \mathrm{O}_{2}, 7 \% \mathrm{CO}_{2}$, and $37{ }^{\circ} \mathrm{C}$ until the embryo was transferred.

\section{Time-lapse monitoring}

Images of each embryo were taken every $20 \mathrm{~min}$ in seven different focal planes, during $72 \mathrm{~h}$ of culture. The embryonic images were analyzed using the Embryo Viewer software (Unisense Fertilitech Aarhus), which annotates embryonic developmental events with the corresponding time (h) after 
microinjection. The times from fertilization to the following events were analyzed: pronuclear fading (Pnf), when both pronuclei disappeared, the first cleavage, when the zygote divides into two cells ( $\mathrm{t} 2$ ), and when cleavage giving rise from 3 to 9 cells was observed for the first time ( $\mathrm{t} 3, \mathrm{t} 4, \mathrm{t} 5, \mathrm{t} 6, \mathrm{t} 7, \mathrm{t} 8$, and $t 9$, respectively). The intervals between two consecutive cleavages were also calculated. The duration of the second cell cycle $(\mathrm{cc} 2=\mathrm{t} 3-\mathrm{t} 2)$ is the time from the division into a two-blastomere embryo until the time to the division into a three-blastomere embryo, and second synchrony $(\mathrm{s} 2=\mathrm{t} 4-\mathrm{t} 3$ ) is the time from this division into a four-blastomere embryo. The duration of the third cell cycle $(\mathrm{cc} 3=\mathrm{t} 5-\mathrm{t} 3)$ is the time to the division into a three-blastomere embryo until the time to division into a five-blastomere embryo. In a previous study, the optimal ranges of the morphokinetic parameters $\mathrm{t} 5$, s2, and cc2 were t $5=48.8-56.6 \mathrm{~h}, \mathrm{~s} 2<0.76 \mathrm{~h}$, and $\mathrm{cc} 2<12 \mathrm{~h}$. In the current study, embryos within these ranges were described as optimal embryos with the highest probability of implantation.

Embryos were graded according to the time-lapse parameters described previously by Basile et al. [16]. The classification was based on the t5-t2 and cc 3 as follows. If the value of the $\mathrm{t} 5-\mathrm{t} 2>20.5 \mathrm{~h}$, the embryo was categorized as A or B; if the value fell outside the optimal range, the embryo was categorized as C or D. If the value of cc3 was between 11 and $18 \mathrm{~h}$, the embryo was categorized as $\mathrm{A}$ or $\mathrm{C}$ and if the value fell outside the optimal range, the embryo was categorized as B or $\mathrm{D}$ depending on the $\mathrm{t} 5-\mathrm{t} 2$ [16].

\section{Embryo transfer}

Embryo transfer was performed on day 3 of development. For luteal support, all patients received $90 \mathrm{mg}$ progesterone gel (Crinone gel 8\%, Merck) and $200 \mathrm{mg}$ vaginal micronized progesterone (Progestan caps 200 mg, Kocak Farma, Turkey) daily. Biochemical pregnancy was established when the serum $\beta$-hCG level was $>20 \mathrm{IU} / \mathrm{I}$ on the 12th day after embryo transfer, and clinical pregnancy was confirmed by ultrasound as the presence of fetal heart activity 8 weeks after embryo transfer.

\section{Follicular fluid sampling}

Individual aspiration was used to collect oocytes, and each follicle was recovered in a different tube. To avoid contamination from blood and flush medium or mixture, only the follicular fluid from the first retrieved follicle, which contains a single oocyte-cumulus complex, was collected. Thus, one follicular sample per patient was used for analysis. Samples of FF were centrifuged at $2000 \times g$ for $10 \mathrm{~min}$ and the supernatants were stored at $-80{ }^{\circ} \mathrm{C}$ for further analysis.

\section{Patient and follicular fluid sample selection process}

A total of 101 patients with unexplained infertility were admitted to the IVF center of Izmir University Medical Park Hospital between November 2015 and March 2016. According to the Turkish Ministry of Health Regulations, single embryo transfer is an obligation for patients under 35 years old in the first two attempts. The patients over 35 years old and patients under 35 years of age who had more than two embryo transfer cycles were questioned for their preference of number of embryo transfer. The patients who prefer more than one embryo transfer $(n=9)$ were not included in the study. Ninety-two patients were prospectively allocated for controlled ovarian hyperstimulation cycle. Patient flow chart is shown in Fig. 1.

The analysis of follicular fluid samples was performed only in patients with single embryo transfer where the embryo was obtained from the oocyte of the sampled follicle. Therefore, follicular fluids of other oocytes were not included and analyzed in the study. Exclusion criteria were absence of mature (MII) oocyte in the aspirated follicle $(n=5)$, fertilization failure $(n=3)$, cleavage arrest $(n=5)$, presence of higher quality embryo for ET other than the one generated from the oocyte coming from the first follicle $(n=11)$, and patients whose embryos were frozen totally for OHSS prevention $(n=5)$ or for the presence of uterine pathologies (myoma $(n=1)$ and endometrial polyp $(n=2)$; Fig. 1). Therefore, the analysis includes only the embryos coming from the first follicle sampled for the concentration of coenzyme Q10 (ug/mL).

\section{Determination of coenzyme Q10 levels in follicular fluid}

Ubiquinone (coenzyme Q10) was determined by reversed-phase high-performance liquid chromatography (HPLC; Shimadzu Prominence, Japan). HPLC kits were purchased from ImmunoChrom $\mathrm{GmbH}$, Heppenheim, Germany. First, high molecular weight substances were removed by centrifugation; then, the supernatant was mixed with an extraction solution and transferred to the organic phase. Following evaporation of the organic solvent, the sample was resuspended in ethanol and injected into the HPLC system. The isocratic separation was performed via HPLC at $30^{\circ}$ $\mathrm{C}$ using a reversed phase column for $15 \mathrm{~min}$. The chromatograms were recorded by a UV detector. The quantification was performed by the delivered calibrator and the concentration was calculated by the internal standard method.

The coefficients of variations were determined by multiple measurement of serum samples. Intra-assay $\mathrm{CV}$ was $0.6 \%$ and inter-assay CV was $6 \%$. The method was linear up to $20 \mu \mathrm{g} /$ $\mathrm{mL}$ and the detection limit was $0.02 \mu \mathrm{g} / \mathrm{mL}$. There was no 
Fig. 1 Patient and follicular fluid selection process
101

patients

92

patients

87

patients

84

patients

79

patients

68

patients

63

patients

60

patients
EXCLUSION

The patients who prefer more than one embryo transfer (n:9)

Absence of mature (MII) oocyte in the aspirated follicle ( $n: 5)$

Fertilization failure ( $n: 3)$

Cleavage arrest (n:5)

Presence of higher quality embryo for ET other than the one generated from the oocyte coming from the first follicle ( $n: 11)$

Patients whose embryos were frozen totally for OHSS prevention (n:5)

Presence of uterine pathologies (myoma (n:1), endometrial polyp (n2).

Follicular fluids were analyzed known cross-reactivity to other quinone-containing compounds.

\section{Statistical analysis}

The SPSS software (SPSS Inc., Chicago, IL, USA) was used for the statistical analysis. Comparisons between two groups were performed with the independent $t$ test for parametric conditions and the Mann-Whitney $U$ test for non-parametric conditions. Pearson and Spearman correlation analysis and receiver operator curves were used. All the values were expressed as the mean \pm standard deviation (SD). A $p$ value $<0.05$ was considered to indicate statistical significance.

\section{Results}

Clinical and laboratory characteristics of pregnant and non-pregnant women are summarized in Table 1 . There was no significant difference between groups in terms of age, body mass index (BMI, $\mathrm{kg} / \mathrm{m}^{2}$ ), duration of infertility, total gonadotropin dose, day 3 serum FSH, serum AMH, and serum estradiol levels on the day of hCG trigger administration. There was no significant difference between groups in terms of retrieved oocytes, MII oocytes, and the number of embryos (Table 1 ).

There was no correlation between the CoQ10 levels with the age of the women, the duration of infertility, total gonadotropin dose, BMI, day $3 \mathrm{FSH}$, estradiol on the day of hCG
Table 1 Baseline characteristics of pregnant and non-pregnant women

\begin{tabular}{lccc}
\hline & Pregnant & Non-pregnant & $p$ \\
\hline Age (years) & $31.82 \pm 4.35$ & $32.56 \pm 4.92$ & 0.546 \\
Infertility duration (years) & $60.60 \pm 39.09$ & $49.50 \pm 32.84$ & 0.333 \\
BMI $\left(\mathrm{kg} / \mathrm{m}^{2}\right)$ & $26.20 \pm 5.99$ & $25.56 \pm 5.21$ & 0.743 \\
AMH $(\mathrm{ng} / \mathrm{mL})$ & $2.04 \pm 1.27$ & $2.07 \pm 1.01$ & 0.927 \\
Day3 FSH $(\mathrm{mIU} / \mathrm{mL})$ & $5.89 \pm 2.20$ & $5.93 \pm 2.06$ & 0.949 \\
E2 $(\mathrm{pg} / \mathrm{mL})$ & $34.96 \pm 10.81$ & $49.10 \pm 52.20$ & 0.201 \\
Total gonadotropin dose $(\mathrm{IU})$ & $2426 \pm 989.94$ & $2506.81 \pm 904.62$ & 0.774 \\
Trigger day E2 & $1743.28 \pm 1113.18$ & $2073.21 \pm 1358.29$ & 0.450 \\
Number of oocytes $(n)$ & $13.29 \pm 8.15$ & $12.95 \pm 5.42$ & 0.871 \\
Number of MII oocytes $(n)$ & $10.58 \pm 6.57$ & $9.63 \pm 4.91$ & 0.586 \\
Number of embryos $(n)$ & $7.79 \pm 5.29$ & $6.81 \pm 3.69$ & 0.477 \\
\hline
\end{tabular}

Data are expressed as mean \pm standard deviation $B M I$ body mass index, $F S H$ follicle stimulating hormone, E2 estradiol $* p<0.05$ 
trigger administration, and AMH. Also, there was no association between CoQ10 levels and morphokinetic parameters including $\mathrm{tPnf}, \mathrm{t} 2, \mathrm{t} 3, \mathrm{t} 4, \mathrm{t} 5, \mathrm{t} 6, \mathrm{t} 7, \mathrm{t} 8$, and $\mathrm{t} 9$.

Follicular fluid CoQ10 levels were significantly higher in grades $\mathrm{A}$ and $\mathrm{B}$ than grades $\mathrm{C}$ and $\mathrm{D}$ embryos $(p<0.05)$. The concentration of follicular fluid CoQ10 levels was significantly higher in the pregnant group $(p<0.05)$. There was no significant relation between optimal $t 5$ and s2 morphokinetic parameters and CoQ10 levels. However, CoQ10 levels were significantly higher in follicular fluid of embryos which had optimal cc2 $(p<0.05$; Table 2).

The area under curve (AUC) of CoQ10 for prediction of pregnancy was 0.677 ( $p=0.022,95 \%$ confidence interval $0.53-0.81$ ). A threshold of 0.255 of CoQ10 had a sensitivity of $68.8 \%$ and specificity of $53.8 \%$. ROC curve of CoQ10 for pregnancy prediction is shown in Fig. 2.

\section{Discussion}

In the present study, we detected a relationship between coenzyme Q10 levels of follicular fluid, optimal embryo morphokinetic parameters, and pregnancy rates. Higher follicular fluid CoQ10 levels are related to better embryo grades and pregnancy rates. The advantages of this study are as follows: first, we could evaluate the CoQ10 levels of a single follicle and later we could investigate the relationship between follicular fluid levels of CoQ10 and that of follicles embryo and later the pregnancy result of this embryo; this is because we had transferred a single embryo to each patient and had evaluated the CoQ10 levels of that follicle. The other advantage of our study was that the oocytes and embryos were evaluated by Embryoscope images continuously and embryo grading was done according to morphokinetic parameters in a more objective way.

Although embryo quality evaluation is mainly determined by morphological evaluation, morphokinetic evaluation has

Table 2 The association between coenzyme Q 10 levels in follicular fluid with embryo morphokinetics and pregnancy results

\begin{tabular}{llll}
\hline & & CoQ10 $(\mathrm{ug} / \mathrm{mL})$ & $p$ \\
\hline t5 & Optimal $(n=23)$ & $0.561 \pm 0.73$ & 0.592 \\
s2 & Non-optimal $(n=36)$ & $0458 \pm 0.70$ & \\
& Optimal $(n=27)$ & $0.521 \pm 0.72$ & 0.825 \\
cc2 & Non-optimal $(n=32)$ & $0.479 \pm 0.71$ & \\
& Optimal $(n=45)$ & $0.572 \pm 0.80$ & $0.013^{*}$ \\
Embryo quality & Non-optimal $(n=14)$ & $0.261 \pm 0.06$ & \\
& Grade A-B $(n=32)$ & $0.526 \pm 0.64$ & $0.038^{*}$ \\
Pregnancy & Grade C-D $(n=15)$ & $0.390 \pm 0.55$ & \\
& Pregnant $(n=26)$ & $0.603 \pm 0.78$ & $0.044^{*}$ \\
& Non-pregnant $(n=32)$ & $0.379 \pm 0.62$ & \\
\hline
\end{tabular}

$* p<0.05$

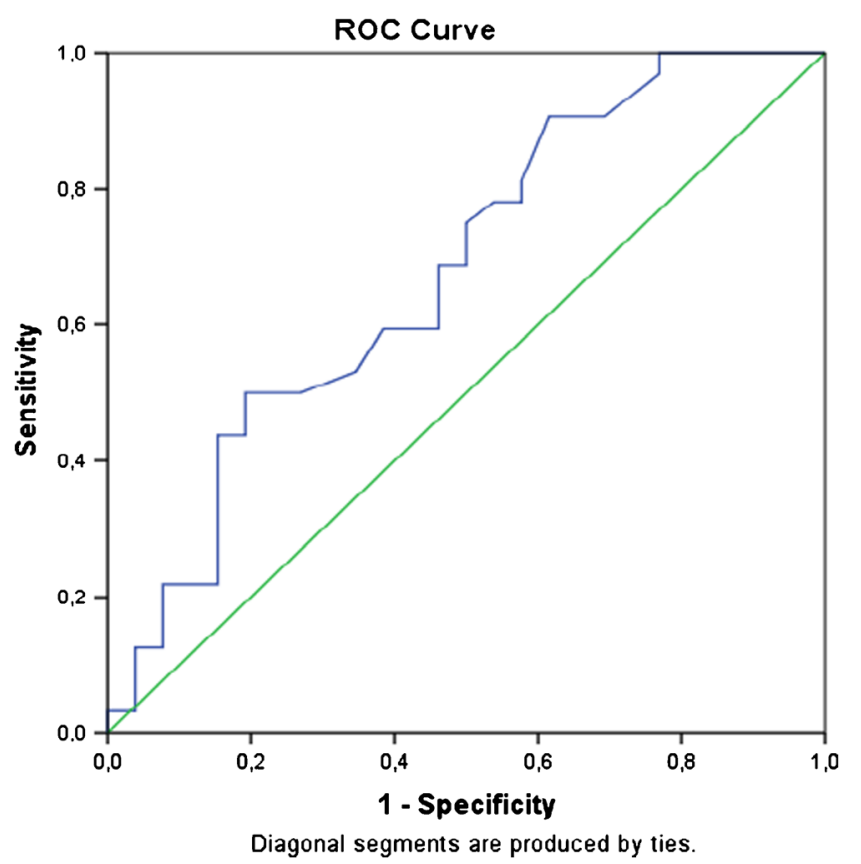

Fig. 2 ROC curve of CoQ10 for the prediction of pregnancy

additional benefits that include the continuous monitoring of embryos, facilitating a more objective and robust information, improved culture conditions, and embryo evaluation without removal and decreased interobserver variability [17, 18]. Also, some recent reports suggested increasing the probability of selecting chromosomally normal embryos by time-lapse morphokinetic analysis [16]. Thus, we preferred to use this algorithm as described by Basile et al. for embryo grading and selecting the transferred embryo in our study.

The intrafollicular environment was suggested to affect the centrosome-based microtubule assembly which might be a factor for the increased aneuploidy rate with age [15]. An imbalance between pro-oxidant and antioxidant defense systems might be related to mitochondrial dysfunction and embryo aneuploidy $[19,20]$. Therefore, increased reactive oxygen species and oxygen radicals should be balanced with antioxidant mechanisms to decrease aneuploidy rates and increase embryo quality [20]. As an electron carrier in mitochondrial bioenergetics and as a lipophilic antioxidant, CoQ10 might have an important role in defending the increased production of reactive oxygen species (ROS) deficiency or susceptibility toward them. Quinzi et al. investigated different levels of CoQ10 deficiency on ROS production and mitochondrial function. Intermediate CoQ10 deficiency was found to be related to a marked increase in ROS production, lipid oxidation, and cell death [21]. CoQ10 treatment was related to less reactive species in the ovaries of mice recently [22]. Also in a recent study, the decrease in CoQ10 concentration in older women was accompanied by a shift in redox status in favor of oxidized form and this effect was attenuated by supplementation with $\mathrm{CoQ} 10$ [23]. 
Another important factor is the energy produced by mitochondria via oxidative phosphorylation that is necessary during oocyte maturation [24]. The bioenergetic state of oocyte was found to be related to the implantation potential of the embryo previously [3]. Any problem during oxidative phosphorylation pathway was reported to lead to the arrest of oocyte maturation, chromosomal misalignment, and compromised embryo development $[5,25,26]$. The key role of CoQ10 in oxidative phosphorylation shows its importance for energy production of oocytes. Thus, Ben-Meir et al. reported that the disruption of CoQ10 synthesis was related to reduced ATP production and increased meiotic spindle abnormalities resulting in infertility in dams [5].

One big question that has been repeatedly posed is whether a CoQ10 concentration of follicular fluid is an important factor for oocyte? And quite sadly, there is limited literature on the subject. Recently, Turi et al. reported that $\mathrm{CoQ} 10 /$ protein levels resulted significantly in mature versus dysmorphic oocytes [27]. Similarly, CoQ10/ cholesterol was significantly higher in grading I-II versus grade III-IV embryos in that study. Our findings are similar to that report. In addition to the aforementioned report, we also found a positive correlation between CoQ10 levels and pregnancy rates. We believe that this result is an important preliminary finding for clinical use of CoQ10 in patients with compromised oocyte maturation and mitochondrial dysfunction. This group of patients cover polycystic ovary syndrome, obesity, diminished ovarian reserve, and advanced age [6, 28]. Thus, recently, Refaeey et al. reported that the combination of CoQ10 and clomiphene citrate improved ovulation and pregnancy rates in patients with polycystic ovary syndrome (PCOS) [28].

The most difficult situations in IVF clinics are patients with diminished ovarian reserve and advanced age. Although CoQ10 is promoted for treating patients' diminished ovarian reserve, there is limited finding on the clinical use of CoQ10 in that group of patients. Clinical studies could not show a significant improvement in pregnancy rates after CoQ10 supplementation in that group of patients [13]. However, CoQ10 concentrations were found to be decreased with advancing age and this decline coincides with a decline in fertility and the increase in embryo aneuploidies [5, 29]. Thus, spontaneous abortions were found to be related with low plasma CoQ10 levels [30]. In the aged animal model, CoQ10 administration improved breeding outcomes, slowed down follicle loss, and improved mitochondrial energetics [5]. According to the results of animal study models, it seems reasonable to use CoQ10 as a mitochondrial micronutrient to alter the aging process [1]. However, the appropriate duration and dosages in humans for reversing the negative impacts on oocytes should be clarified. Thus, the use of CoQ10 for 12-16 weeks in a mouse was reported to be equivalent to about a decade in human [5].
In conclusion, we found a positive correlation between follicular fluid CoQ10 concentrations and subsequent embryo quality and pregnancy rates. Our results might be supportive of the usage of CoQ10 as a supplement in women undergoing in vitro fertilization. Further studies that will involve a larger, randomized, and controlled group are needed to draw definitive conclusions for the clinical administration of CoQ10 in female infertility.

\section{References}

1. Bentov Y, Casper RF. The aging oocyte - can mitochondrial function be improved? Fertil Steril. 2013;99(1):18-22.

2. Trifunovic A, Wredenberg A, Falkenberg M, Spelbrink JN, Rovio AT, Bruder CE, et al. Premature ageing in mice expressing defective mitochondrial DNA polymerase. Nature. 2004;429:417-23.

3. Van Blerkom J, Davis PW, Lee J. ATP content of human oocytes and development potential and outcome after in-vitro fertilization and embryo transfer. Hum Reprod. 1995;10:415-24.

4. Wilding M, Dale B, Marino M, Matteo L, Alviggi C, Pisaturo ML, et al. Mitochondrial aggregation patterns and activity in human oocytes and preimplantation embryos. Hum Reprod 2001; 16: 909-917.

5. Ben-Meir A, Burstein E, Borrego-Alvarez A, Chong J, Wong E, Yavorska T, et al. Coenzyme Q10 restores oocyte mitochondrial function and fertility during reproductive aging. Aging Cell 2015: $887-895$.

6. May Panloup P, Chretien MF, Malthiery Y, Reynier P. Mitochondrial DNA in the oocyte and the developing embryo. Curr Top Dev Biol. 2007;77:51-83.

7. Gendelman $\mathrm{M}$, Roth Z. Incorporation of coenzyme Q10 into bovine oocytes improves mitochondrial features and alleviates the effects of summer thermal stress on developmental competence. Biol Reprod. 2012;87(5):118,1-12.

8. Stojkovic M, Westesn K, Zakhartchenko V, Stojkovic P, Boxhammer K, Wolf E. Coenzyme Q10 in submicron-sized dispersion improves development, hatching, cell proliferation, and adenosine triphosphate content of in vitro-produced bovine embryos. Biol Reprod. 1999;61:541-7.

9. Garrido-Maraver J, Cordero MD, Oropesa-Avila M, Vega AF, La Mata M, Pavon AD, et al. Coenzyme Q10 therapy. Mol Syndromol. 2014;5:187-97.

10. Mancini A, De Marinis L, Oradei A, Littaru GP, Balercia G. An update of coenzyme Q10 implications in male infertility: biochemical and therapeutic aspects. Biofactors. 2005;25:165-74.

11. Safarinejad MR. The effect of coenzyme Q10 supplementation on partner pregnancy rate in infertile men with idiopathic oligoasthenoteratozoospermia: an open-label prospective study. Int Urol Nephrol. 2012;44:689-700.

12. Bentov Y, Hannam T, Jurisicova A, Esfandiari N, Casper RF. Coenzyme Q10 supplementation and oocyte aneuploidy in women undergoing IVF-ICSI treatment. Clin Med Insights Reprod Health. 2014;8:31-6.

13. Gat I, Mejia SB, Balakier H, Librach CL, Claessens A, Ryan EAJ. The use of coenzyme Q10 and DHEA during IUI and IVF cycles in patients with decreased ovarian reserve. 2016; 32(7):534-537.

14. Revelli A, Piane LD, Casano S, Molinari E, Massobrio M, Rinaudo P. Follicular fluid content and oocyte quality: from single biochemical markers to metabolomics. Reprod Biol Endocrinol. 2009;7:40. 
15. Dumesic DA, Meldrum DR, Katz-Jaffe MG, Krisher R, Schoolcraft WB. Oocyte environment: follicular fluid and cumulus cells are critical for oocyte health. Fertil Steril. 2015;103:303-16.

16. Basile N, Nogales MC, Bronet F, Florensa M, Riqueiros M, Rodrigo L, et al. Increasing the probability of selecting chromosomally normal embryos by time-lapse morphokinetic analysis. Fertil Steril. 2014;101:699-704.

17. Aparicio B, Cruz M, Meseguer M. Is morphokinetic analysis the answer? Reprod Biomed Online. 2013;27:654-63.

18. Sundvall L, Ingerslev HJ, Breth Knudsen U, Kirkegaard K. Interand intra-observer variability of time-lapse annotations. Hum Reprod. 2013;28:3215-21.

19. Agarwal A, Aponte-Mellado A, Premkumar BJ, Shaman A, Gupta $\mathrm{S}$. The effects of oxidative stress on female reproduction: a review. Reprod Biol Endocrinol. 2012;10:49.

20. Devine PJ, Perreault SD, Luderer U. Roles of reactive oxygen species and antioxidants in ovarian toxicity. Biol Reprod. 2012;86:27.

21. Quinzii CM, Tadesse S, Naini A, Hirano M. Effects of inhibiting CoQ10 biosynthesis with 4-nitrobenzoate in human fibroblasts. PLoS One. 2012; 7:e30606.

22. Luce K, Weil AC, Osiewacz HD. Mitochondrial protein quality control systems in aging and disease. Adv Exp Med Biol. 2010;694:108-25.

23. Niklowitz P, Onur S, Fischer A, Laudes M, Palussen M, Menke T, et al. Coenzyme Q10 serum concentration and redox status in
European adults: influence of age, sex and lipoprotein concentration. J Clin Biochem Nutr. 2016;58:240-5.

24. Dumollard R, Ward Z, Caroll J, Duchen MR. Regulation of redox metabolism in the mouse oocyte and embryo. Development. 2007; 134:455-65.

25. Takeuchi T, Neri QV, Katagiri Y, Rosenwaks Z, Palermo GD. Effect of treating induced mitochondrial damage on embryonic development and epigenesist. Biol Reprod. 2005;72:584-92.

26. Thouas GA, Trounson AO, Jones GM. Developmental effects of sublethal mitochondrial injury in mouse oocytes. Biol Reprod. 2006;74:969-77.

27. Turi A, Giannubilo SR, Bruge F, Principi F, Battistoni S, Santoni F, et al. Coenzyme Q10 content in follicular fluid and its relationship with oocyte fertilization and embryo grading. Arch Gynecol Obstet. 2012;285:1173-6.

28. Refaeey AE, Selem A, Badawy A. Combined coenzyme Q10 and clomiphene citrate for ovulation induction in clomiphene-citrateresistant polycystic ovary syndrome. Reprod Biomed Online. 2014;29:119-24.

29. Miles MV, Horn PS, Tang PH, Morrison JA, Miles L, DeGrauw T, et al. Age related changes in plasma coenzyme Q10 concentrations and redox state in apparently healthy children and adults. Clin Chim Acta. 2004;347:139-44.

30. Noia G, Littaru GP, De Santis M, Oradei A, Mactromarino C, Trivellini C, et al. Coenzyme Q10 in pregnancy. Fetal Diagn Ther. 1996;11:264-70. 